\title{
Lessons Learned during Instrument Testing for the Thermal Infrared Sensor (TIRS)
}

\author{
Hume L. Peabody ${ }^{1}$, Veronica Otero ${ }^{2}$ \\ NASA Goddard Space Flight Center, Greenbelt MD 20771 \\ and \\ David Neuberger ${ }^{3}$ \\ Edge Space Systems, Glenelg MD 21737
}

\begin{abstract}
The Themal InfraRed Sensor (TIRS) instrument, set to launch on the Landsat Data Continuity Mission in 2013, features a passively cooled telescope and IR detectors which are actively cooled by a two stage cryocooler. In order to proceed to the instrument level test campaign, at least one full functional test was required, necessitating a thermal vacuum test to sufficiently cool the detectors and demonstrate performance. This was fairly unique in that this test occurred before the Pre Environmental Review, but yielded significant knowledge gains before the planned instrument level test. During the pre-PER test, numerous discrepancies were found between the model and the actual hardware, which were revealed by poor correlation between model predictions and test data. With the inclusion of pseudo-balance points, the tést also provided an opportunity to perform a pre-correlation to test data prior to the instrument level test campaign. Various lessons were learned during this test related to modeling and design of both the flight hardware and the Ground Support Equipment and test setup. The lessons learned in the pre-PER test resulted in a better test setup for the nstrument level test and the completion of the final instrument model correlation in a shorter period of time. Upon completion of the correlation, the flight predictions were generated including the full suite of off-nominal cases, including some new cases defined by the spacecraft. For some of these new cases, some components now revealed limit exceedances, in particular for a portion of the hardware that could not be tested due to its size and chamber limitations. Further lessons were learned during the completion of flight predictions. With a correlated detailed instrument model, significant efforts were made to generate a reduced model suitable for observatory level analyses. This proved a major effort both to generate an appropriate network as well as to convert to the final model to the required format and yielded additional lessons learned. In spite of all the challenges encountered by TIRS, the instrument was successfully delivered to the spacecraft and will soon be tested at observatory level in preparation for a successful mission launch.
\end{abstract}

$\begin{array}{ll}A P G & =\text { Annealed Pyrolytic Graphite } \\ B B C A L & =\text { Black Body Calibrator } \\ C C E & =\text { CryoCooler Electronics } \\ C C H P & =\text { Constant Conductance Heat Pipe } \\ C P U & =\text { Central Processing Unit } \\ F P E & =\text { Focal Plane Electronics } \\ G S E & =\text { Ground Support Equipment } \\ G S F C & =\text { Goddard Space Flight Center } \\ L N 2 & =\text { Liquid Nitrogen } \\ L D C M & =\text { Landsat Data Continuity Mission }\end{array}$

\section{Nomenclature}

$M E B=$ Main Electronics Box

$M L I \quad=$ Multi-Layer Insulation

$N A S A=$ National Aeronautics and Space Administration

$O L I=$ Operational Land Imager

$P C=$ Personal Computer

$P E R$ = Pre Environmental Review

SINDA $=$ Systems Improved Numerical Difference Analyzer

$S S M=$ Scene Select Mechanism

TIRS = Thermal InfraRed Sensor

$T V \quad=$ Thermal Vacuum

\footnotetext{
${ }^{1}$ Staff Thermal Engineer, GSFC-Code 545.0, Greenbelt, MD 20771

${ }^{2}$ Associate Branch Head, GSFC-Code 545.0, Greenbelt, MD 20771

${ }^{3}$ Thermal Engineer, Edge Space Systems, Glenelg, MD 21737
} 


\section{Introduction}

$\mathrm{T}$ he Thermal InfraRed Sensor (TIRS) is an IR sensing instrument on the Landsat Data Continuity Mission (LDCM) set for an early 2013 launch. LDCM flies in a low Earth oriented orbit ranging in altitude from $704 \mathrm{~km}$ to $728 \mathrm{~km}$ with beta angles ranging from $+20^{\circ}$ to $+38^{\circ}$. LDCM has a mission design life of 3.5 years and supports a payload of two science instruments, TIRS, and the Operational Land Imager (OLI) as depicted in Figure 1. The TIRS instrument was designed, built, and tested at the Goddard Space Flight Center (GSFC). TIRS utilizes a 2 stage cryocooler to cool the detectors to $38 \mathrm{~K}$ to reduce the signal to noise ratio. As such, it was not possible to conduct a full functional test in ambient conditions prior to the Pre Environmental Review (PER), which generally signifies the beginning of the instrument level test campaign. Therefore, a PrePER thermal vacuum test with a full functional was performed before the traditional PER; this test yielded significant lessons learned which resulted in design modifications for the test setup for the instrument level test as well as providing early test data to begin model correlation efforts prior to PER. The TIRS project was also operating under intense schedule pressure, with a delay

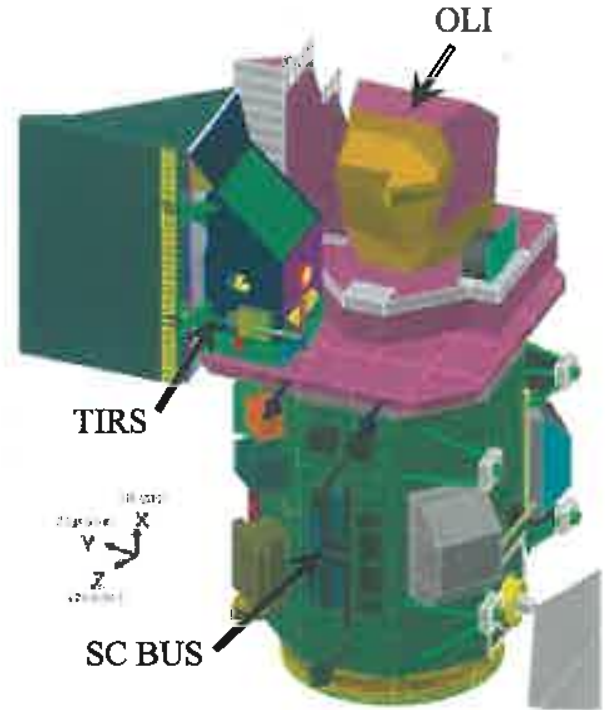

Figure 1. LDCM Thermal Model TIRS and OLI on Instrument deck

in the funding profile at the start but a fixed delivery date to the spacecraft. At times, this resulted in a hurried process and insufficient time to ensure that model was updated to reflect the as-built hardware prior to testing. In spite of all the challenges the team faced, TIRS was successfully delivered to the spacecraft vendor for integration in March of 2012 and successfully launched on February 11, 2013.

\section{TIRS Thermal Design}

TIRS features a scene select mirror which redirects incident energy (nominally from a nadir view in the $+Z$ direction) through a telescope onto the detectors. The Scene Select Mechanism (SSM) rotates the scene select mirror to redirect the view to one of three locations: through the nadir aperture for science data collection, through the space aperture on the continuously cold $+Y$ face for a deep space calibration, or to an internal temperature controlled blackbody calibrator for a hot calibration. The telescope itself is passively cooled via a high conductivity thermal strap to a dedicated radiator facing in the $+\mathrm{Y}$ direction and is thermally isolated from the support structure. The detectors are cooled by a 2 stage cryocooler, with the first stage providing pre-cooling to $105 \mathrm{~K}$ and the second stage providing cooling to $38 \mathrm{~K}$ for the detectors. The power generated by the cryocooler is transferred via heat pipes to a dedicated radiator also facing in the $+Y$ direction. The Focal Plane Electronics (FPE) box, which controls the detectors, is mounted to but thermally isolated from, the $+Z$ structural panel. The top of the FPE chassis is used as a nadir facing radiator to maintain the temperatures within the performance range. Various operational heaters control and maintain stability for the warm stage, the telescope, the Scene Select Mechanism (SSM), the Focal Plane

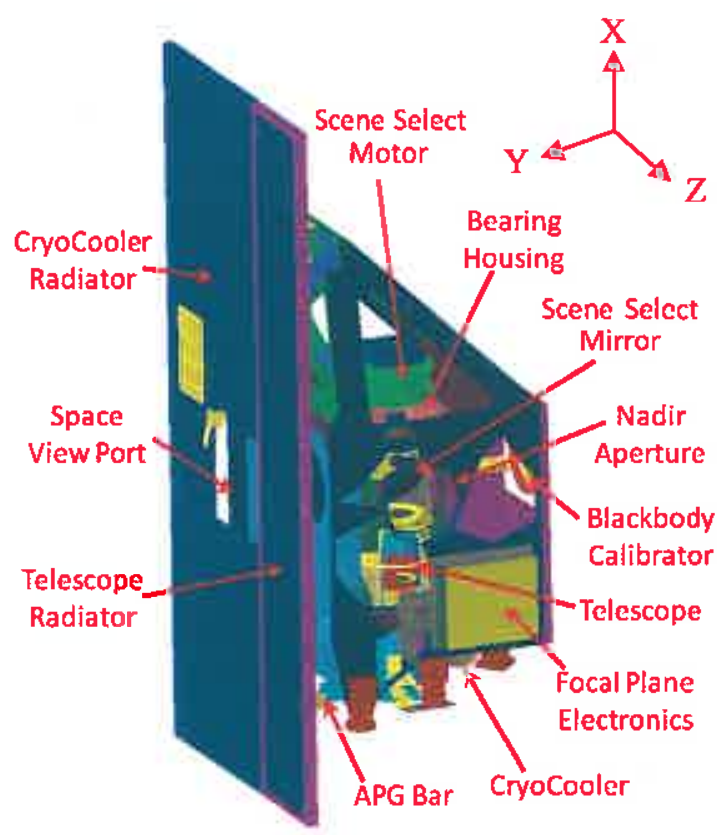

Figure 2. TIRS Thermal Design Key components of TIRS Thermal Design (EarthShield and MLI not shown) Electronics (FPE), and the Blackbody Calibrator (BBCAL). The instrument itself is thermally isolated from the spacecraft mounting deck through low conductivity flexures. Two additional electronics boxes, the Main Electronics Box (MEB) and the CryoCooler Electronics (CCE), are used to control the instrument and the cryocooler, but they are mounted away from the TIRS sensor unit and under spacecraft thermal control responsibility. 


\section{TIRS TV Testing}

The TIRS Pre-PER thermal testing was conducted from August 2011 through September 2011 at GSFC. Due to chamber constraints, TIRS was tested without the EarthShield. Furthermore, the orientation of the calibration equipment did not allow for the instrument to be tested in an orientation where the heatpipes were level, but the main transport pipes were oriented such that they would operate in reflux mode. The spreader heatpipes in the lower portion of the cryocooler radiator did not function in the test orientation, while the pipes in the upper portion operated in reflux mode. The calibration equipment was all controlled to LN2 temperature to minimize the background thermal noise. The MEB and CCE were mounted on cold plates and controlled by their respective baseplate temperatures. The sensor unit itself was bolted to a temperature

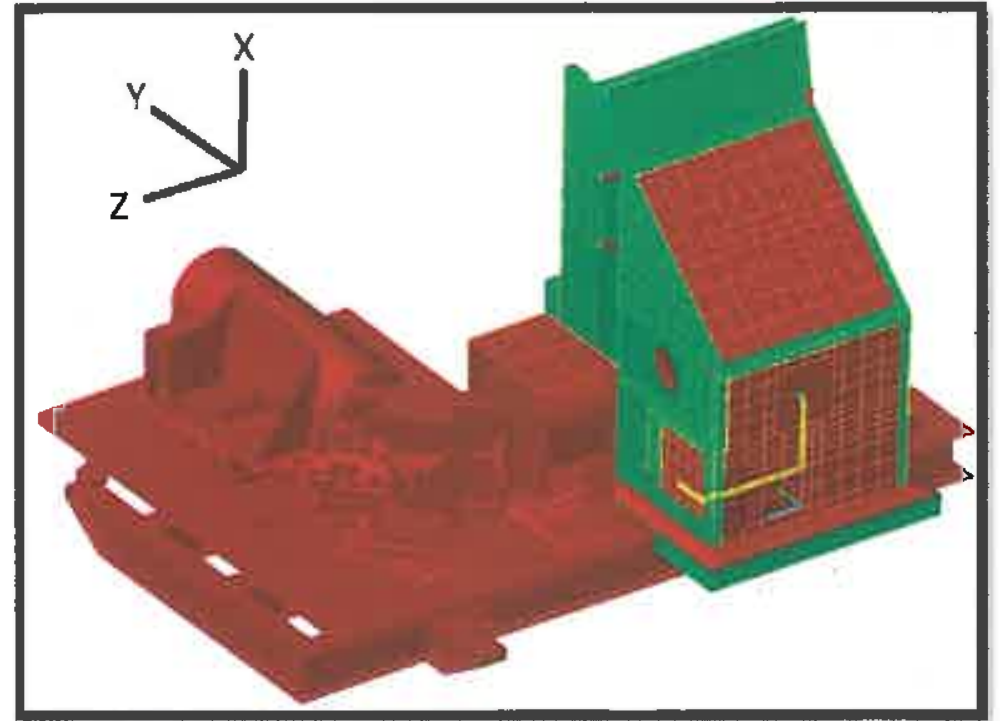

Figure 3. TIRS TV Test Configuration view of entire test setup including Calibration Equipment and GSE enclosing Instrument. Blanketing shown in Green; Red is unblanketed. controlled spacecraft interface plate and surrounded by heater panels or cryopanels to control the temperature sink on all sides. Two cryopanels, controlled with LN2, provided the sink for the two +Y radiators (CryoCooler and Telescope) with a small blanket separating the views of each radiator to its respective cryopanel. The remaining sides were controlled using heater panels with one zone for the $-Z$ (zenith), one for the $+X$, one for $-Y$, and three for the $+Z$ (nadir) side. The middle heater panel on the $+Z$ side featured an aperture to allow the calibration equipment signals into the instrument. A separate panel on the $-Y$ side provides the sink for the BBCAL radiator. Heat straps from the $+Z$ heater panels and from the BBCAL heater plate on the $-Y$ side to an LN2 cold plate provide the cold biasing; all other heater panels rely on radiation to the chamber shroud for cold biasing. Lastly, a temperature controlled plate, known as the Earthshield Stub, was bolted to the Earthshield/Hinge structure to simulate the heat leak from the EarthShield during testing. The testing setup is shown in Figure 3. The instrument itself, without the EarthShield is shown in Figure 4 as well as the layout of Kapton heaters on the heater panels.
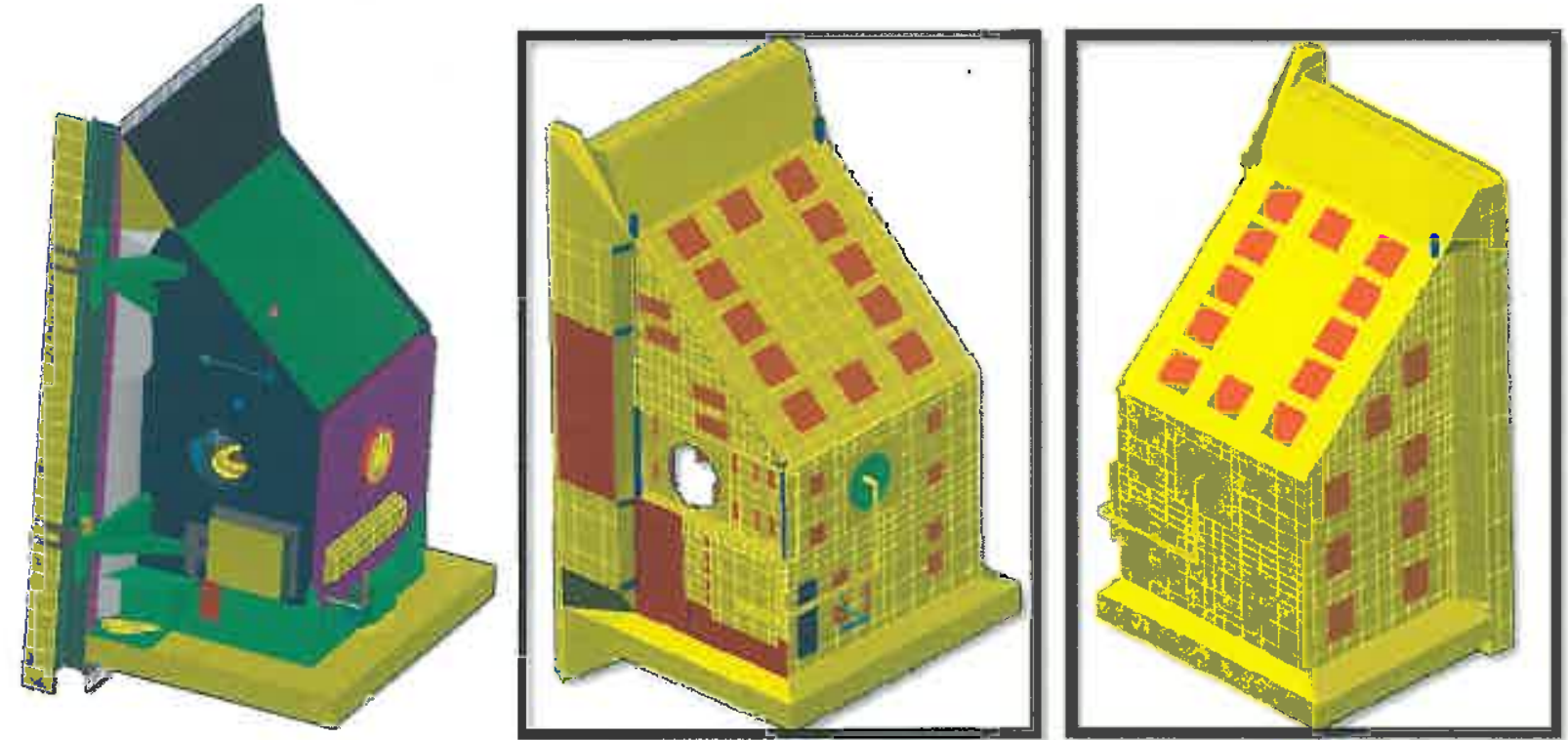

Figure 4. TIRS TV Test Configuration Instrument test article with no EarthShield (Left) and Heater Layout on surrounding Heater Panels (Middle and Right) 


\section{TIRS TV Testing Setup Lessons Learned}

The final test configuration evolved from the PrePER test. Changes were made in the MLI, test heat straps to cold plates, and flight blanketing. The pretest model very coarsely nodalized the heater panels, with only 2-3 nodes representing an entire panel. These panels were nominatly $0.060^{\prime \prime}$ to $0.090^{\prime}$ thick and were partially blanketed in the Pre-PER test, as shown in Figure 5, to conserve test heater power. This partial blanketing and lack of thickness produced considerable gradients from heater locations to bolt locations to the supporting framework. For the instrument level test, this partial blanketing was removed and additional heater circuits used to provide for a more uniform temperature distribution on the panels. Furthermore, the lack of nodalization in the original model as shown in Figure 5 made it impossible to accurately apply test boundary condition, either in terms of temperature sensors or applied heater
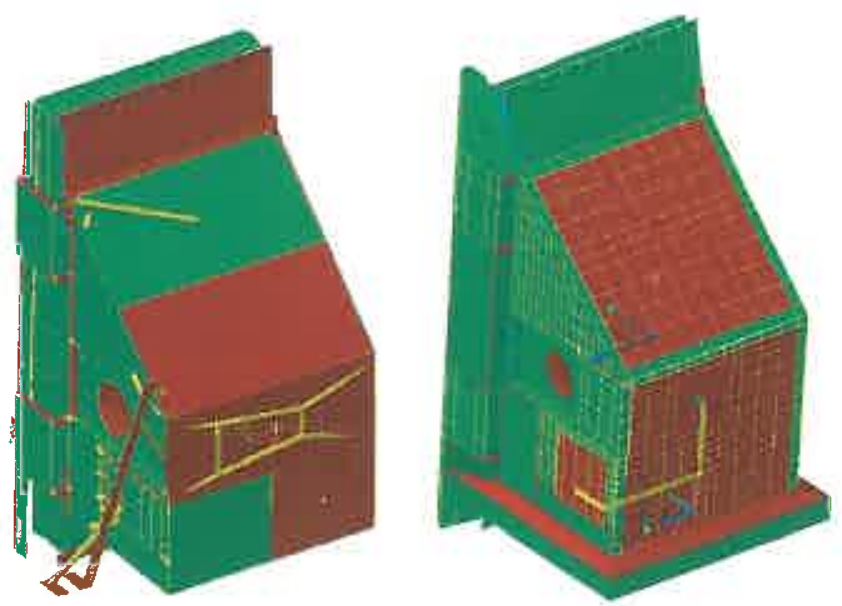

Figure 5. TIRS TV GSE Model Pre-PER Test Nodalization and Blanketing (Left) vs. Instrument Level Test Nodalization and Blanketing (Right) - Red is No Blanket, Green is Blanket power due to the large gradients and insufficient nodes to capture this behavior.

Lesson Learned: Be careful if partially blanketing heater panels as this could induce large gradients for thin panels. Careful pre-test analysis should be used to determine an appropriate level of nodalization, optimum thickness, blanketing, and reasonable heater layout and placement to minimize gradients in the panel.

Since the two main $+Y$ radiators operate at vastly different temperatures, with the telescope nominally at $170 \mathrm{~K}$ and the cryocooler nominally at $270 \mathrm{~K}$, there were concerns with radiative crosstalk if a single zone was used. To minimize this cross talk, a closeout blanket was added between two zones (shown in purple in Figure 6). Furthermore, the red portion in Figure 6 represents an intermediate heater panel used to provide trim heating of the sink for the cryocooler radiator.

Unfortunately, this closeout internal blanket was very difficult to install due to minimal access between the radiators and the cryopanels. During test execution, the telescope radiator was warmer than predicted and sources of additional heat leaks were investigated. Upon completion of the PrePER test, the blanket was investigated and was found to have a significant twist, which would allow the warm cryocooler radiator to see the telescope radiator via reflections off the cryopanel. A photograph of this closeout is shown in Figure 6 along with labels for key components.

Lesson Learned: Unsupported internal closeout blankets can be very difficult to maneuver into position and ensure that they do not move. If possible, provide a means of mechanically attaching the blanket and ensure the finished assembly is inspected.
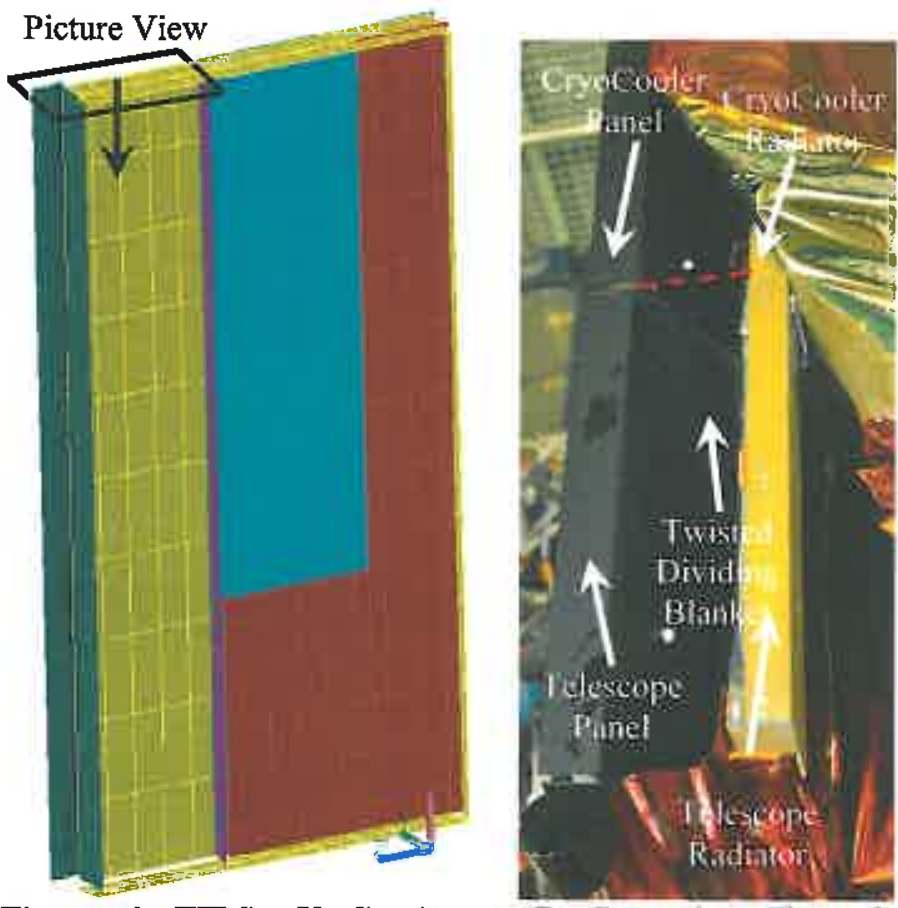

Figure 6. TIRS $+Y$ CryoPanel Configuration Thermal model representation Telescope Radiator CryoPanel (YellowLeft), Internal Closeout Blanket (Purple-Left), CryoCooler Radiator CryoPanel (Blue-Lefi) and HeaterPanel (Red-Left). Photograph of Internal Closeout Blanket (Right) Dashed red line is where it should have been.

4 
Due to the compressed schedule for TIRS to meet the LDCM launch date, significant conservatism was used to size the cryocooler radiator, especially since this particular cryocooler did not have flight heritage on which to base the expected dissipations, and testing of the flight unit occurred very late in the schedule. The cryocooler radiator was therefore sized for a worst case dissipation of $180 \mathrm{~W}$. With this high of a load, it was expected that the LN2 cryopanel would be insufficient to achieve the desired cold qualification temperatures radiatively, especially with some of the spreader heat pipes non-functioning in the vertical orientation used in test. Therefore, a strap was designed between the cryocooler cryopanel and the back side of the cryocooler radiator itself. A heater on the strap mount on the radiator side was used to make this interface adiabatic by applying sufficient heat to compensate for the heat loss through the strap and not introduce additional heat beyond the intended design. For cold qualification, this heater could be adjusted to allow for additional conductive cooling to achieve qualification temperatures on the cryocooler radaitor. In the end, the measured cryocooler power during the test $(71 \mathrm{~W}$ at Hot Balance and $87 \mathrm{~W}$ at Hot Qualification) was significantly less than the $180 \mathrm{~W}$ for which the radiator was sized. The subsequent instrument level test abandoned this strap since the qualification temperatures could be radiatively achieved with the diminished power.

Lesson Learned: Uncertainty in power dissipations can lead to a more complex test design that may be unnecessary. Projects should strongly consider the impacts on the entire program of simply carrying large design margins instead of ensuring that power dissipations can be measured within a reasonable time frame.

Lastly, for the Pre-PER test, the BBCAL radiator GSE panel relied on radiation to the cold shroud for cold biasing, providing the temperature sink for the heater controlled black body. The analysis showed it should be sufficient to cold bias radiatively, but it did not indicate healthy margin. During the Pre-PER test, the cold temperatures could not be achieved to sufficiently validate that enough heater power was available to maintain the desired hot temperatures of the blackbody. For the subsequent instrument level test, a heat strap to a cold plate was implemented to provide the cold biasing rather than relying on radiation alone.

Lesson Learned: For aspects of a design where control authority is to be demonstrated by testing, it is recommended to conductively cold bias the temperature sink rather than relying on radiation and analysis, especially if the radiative cooling capability may be marginal.

\section{TIRS TV Analysis Preparation Lessons Learned}

The model correlation after each TV test took considerable time and effort and uncovered numerous modeling inaccuracies along the way due to the compressed schedule available for completion of the model. Sometimes it is impossible to know in advance where modeling inaccuracies lie, until the data reveals them. But other times a thorough scrub of the model by knowledgeable personnel (as well as independent reviewers) and comparison to mechanical models can reveal errors prior to discovering them through differences between test data and predictions. While project schedules may be very aggressive, eventually this level of scrutiny and attention to detail must be reconciled. For TIRS, many of the adjustments made during correlation were simply ensuring that the model accurately represented the as built hardware and was less a product of parameter uncertainty. Furthermore, the link between the model output parameters and the test data parameters to be extracted for correlation comparison was not firmly established prior to the test. Therefore, the subset of data for comparison grew from the initial subset prior to pumpdown to later points in the test.

\section{A. Pre-Test Model Audit}

Leading up to the test, most of the subsystems in the thermal model underwent a thorough review. The necessary updates were identified, but through the aggressive schedule, a breakdown occurred and many of the updates were not implemented before the test pumpdown. A sudden and unexpected departure of the primary TIRS analyst before the analysis was completed and just before the start of the test further exacerbated the disconnects between the actual hardware and the analytical model. This necessitated a new analyst having to take over modeling responsibilities without a planned handover or transition period. The model was delivered in its current state without documentation as to what areas identified in the model audit still needed updating. Early results from the test showed some significant deviations from the predictions, resulting in a duplication of the model audit process, but this time in real time as the test was underway.

During the correlation process as the test progressed, specific attention was given to each subsystem of the overall instrument with careful detail given to interfaces, thicknesses, model repreșentations, and materials. The sizes of many parts of the Scene Select Mechanism assembly were incorrect compared to the solid model and the actual 
hardware as wcll and some incorrect material assignments were made for the motor shaft. While the external view may have looked "close enough" in the model, the larger size in the thermal model of the Fixed and Rotating Baffles provided considerably more radiating area than the actual hardware. Also, the actual effective radiator area was not measured for the FPE, with the area being larger in the model compared against actual hardware due to differences in the blanketing represented in thermal model. During the test, additional time was spent to troubleshoot this discrepancy, which could have been avoided if the actual radiator size was known and implemented in the model prior to the test. Lastly, comparing the masses of the thermal model to that of the mechanical model and mass properties reports also showed some major discrepancies, often due to the inclusion of inserts, fasteners and such, which impacted the transient response. This region was addressed after test completion when flight predictions showed some larger than expected orbital temperature swings, likely due to the under representation of the overall mass of the components. The availability of a complete mass tree from the Mechanical subsystem aided in the effort, but would have been helpful if it had been completed and made available earlier.

Unfortunately, the very aggressive schedule and the pressure to get the test underway did not allow time for the model to be synchronized with the hardware before the test; however during the test, with efforts to correlate the model, a thorough model scrub was performed in real time and under greater pressure during the test. With many changes between design reviews and actual as built hardware, projects should allow sufficient time for this kind of review prior to testing. Once the door closes, the ability to compare model and hardware is greatly diminished.

Lesson Learned: Prior to testing, a thorough audit of a model should be performed by personnel (not just thermal) who are intimately familiar with the design and hardware to detect any discrepancies that could produce spurious predictions. This audit should include a mass check, size and dimension check, interface check, and actual radiator size verification. Model updates should be completed prior to beginning the test.

\section{B. Pre-Test Data Audit}

The data to be compared between model and the test article was also not clearly defined ahead of the test. As such, early correlation predictions did not include comparisons to all the data points of later ones as additional data points were added to the comparison as the test progressed. If a template had been developed prior to the test of all the thermal related data of interest, then a complete set could have been produced for all intermediate model updates. Furthermore, the model itself was not originally configured to easily produce the points of interest. As the test was running, additional data points from the model were being added using Measures in ThermalDesktop to produce the temperatures at specific telemetry locations. At the same time, updates were being made to the model to improve the correlation at locations with the largest discrepancies. This resulted in a disconnect between results produced with earlier and later versions of the model when comparing to test data. If the model had been configured prior to the test for all the output needed, the results would have been easier to compare between iterations of the model.

Lesson Learned: Prior to testing, a template should be developed with all the data parameters to be compared between model and test article (Flight Hardware Dissipations and Temperatures, GSE Dissipation and Temperatures, Configuration States, Heater Setpoints, etc). The model should also be configured to output all of the test data points for comparison. Doing this ahead of time will allow for the identification of gaps in the model or test data to be filled prior to the availability of the test as the test progresses.

\section{Pre-Correlation Check}

With the schedule pressure, correlation of the model began as soon as quasi-steady test data was available and continued in parallel with the testing. The model was updated often during this time to better correlate to the first hot balance point. Numerous changes were made to both the GSE representations as well as the flight model, including the updates to the as measured dissipations and test data for the surrounding GSE temperatures. By the time the cold balance point was reached, some days later, the model had evolved considerably from original test model making it very difficult to compare the pre-test predictions to the same model, but with only the GSE environments and power dissipations updated. This made it difficult to answer the question of "How far were we off from the pre-test values?" since the model had evolved considerably in that time span.

Lesson Learned: Prior to making any changes to the baseline pre-test model, generate a model with only the known values updated, such as power dissipation, GSE settings and temperatures. However, as a corollary to this recommendation, the baseline model should be configured prior to the test to be ready to accept these inputs without needing further modification. This same model should then be compared for Cold Balance and Hot Balance conditions to establish the pre-test model errors. 


\section{TIRS TV Analysis Techniques Lessons Learned}

The TIRS model was developed and maintained in ThermalDesktop v5.4. Some of the modeling practices in the TIRS model were less than optimal including: over-culling of radiation couplings, shorting of nodes with a high conductor, lack of organization of entities by submodel, enabling or disabling of entities through symbols, and others. With the sudden and unexpected departure of the primary TIRS analyst before the analysis was completed, a new analyst took over modeling responsibilities with little model documentation on which to rely. The original test model was transferred to GSFC at the start of the TV test and required considerable effort to navigate the model structure. The first major update was to update the coarse nodalization of the GSE and replace it with a finer resolution representation since the heater panels surrounding the instrument showed considerable gradients and the heaters did not cover the entire footprint of the panels. Once a better representation of the GSE was established, the model itself was investigated beginning with areas with the regions having the largest temperature discrepancies.

During comparison of the detailed and reduced models, some discrepancies were identified in the components with views to the telescope radiator. After further investigation and validation of the discrepancy, the root cause was traced back to the culling of radiation couplings by the two models. The reduced model included all radiation couplings, regardless of magnitude. The detailed model included only couplings above a $B_{i j}$ of 0.001 with a $B_{i j}$ sum of 0.95 to minimize the total number of conductors to be included in the thermal model. However, this neglected some small couplings for which the temperature difference could result in non-negligible heat flows, even though the radiative coupling appeared negligible. While the affected couplings did not significantly impact the overall temperature predictions, it did call into question the behavior between the modeis and should be deliberately verified before settling on the culling terms.

Lesson Learned: For cryogenic or near cryogenic designs, careful attention should be paid to the radiation couplings that are neglected as they could have importance if the temperature difference is sufficient.

As a means of model connectivity, numerous areas were coupled together with a contactor or conductor using a very high conductance value. While this works in theory to "merge" two nodes, the impact on the numerical solution algorithm for iterative solutions far outweighs the time saved by "connecting" rather than renumbering the necessary nodes in ThermalDesktop. When a very large coupling is used, it also artificially increases the CSGMN (Capacitance divided by Sum of Conductors) term which is a measure of allowable timesteps for transient solutions and may limit the use of more efficient automatic timestep determination by the software. It also hampers convergence since the solution of the nodal temperature iteratively depends on whether the very strongly coupled node has or has not been previously solved in this iteration and can cause oscillations in the solution, preventing convergence. In general, this practice should be avoided.

Lesson Learned: Never couple two nodes with a very high coupling in order to make them have the same temperature Renumber the node or merge them instead as this generates a far more robust thermal network for solution algorithms.

This same high coupling technique was incorrectly applied to a particular area of the model and ignored the physics of heat flow. A high in-plane conductivity APG bar connected the telescope radiator to the telescope cryo shell. The end of this bar formed a " $T$ " shape to which the top part of the "T" was coupled to two ethane CCHPs (as shown in Figure 7). The vertical part of the "T" was modeled using a high conductivity contactor to the top part of the "T". This top part of the "T" was modeled as an edge node, $2 \times 2 \times 4$ solid brick. This approach ignored the in plane resistance in the top portion of the " $\mathrm{T}$ " by not including sufficient nodalization along the heat path and shorted the APG bar to the heat pipes by neglecting resistance.

Lesson Learned: For cryogenic designs in particular, it is extremely important to include all sources of resistance in a heat path to avoid "shorting" sections which could result in overly optimistic predictions.
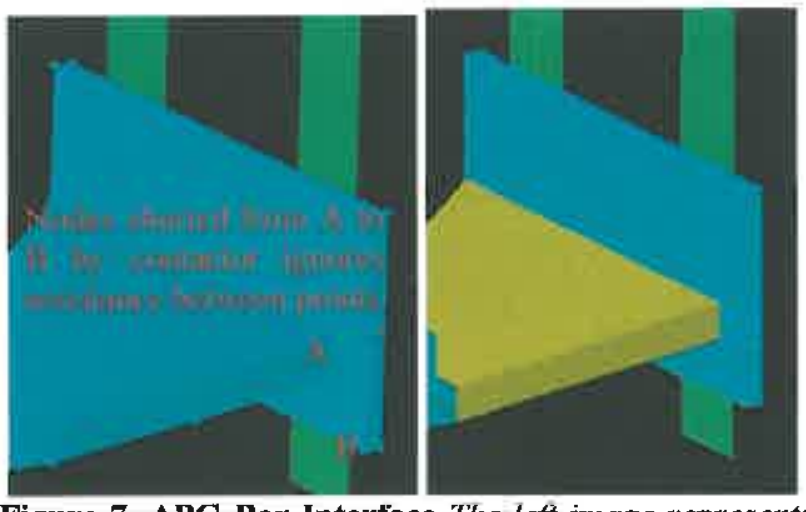

Figure 7. APG Bar Interface The left image represents the nodalization prior to correlation, where the $T$ interface shorted the nodes to the corners with a $99 \mathrm{~W} / \mathrm{K}$ contactor; the right image includes the resistance through the material to interface to the heat pipes shown in green with nodes properly merged

American Institute of Aeronautics and Astronautics 
Model organization is very important, especially in a model as complex as TIRS. With more and more of the model being autonomously generated by current generations of software, logical organization is highly advantageous for the analysts, but even more so when the model is handed off to other analysts or delivered to a higher level of assembly. The TIRS model included a large number of the contactors in the MAIN submodel, even though there were no solution nodes in this submodel. This made it difficult to gain a full understanding of the model and its connectivity for a new analyst since there was no connection between the nodes/submodels connected by the contactor and the submodel to which the contactor was assigned. While the inclusion of descriptions was helpful, better organization by relevant submodel would have improved the navigation of the model.

Lesson Learned: For the more complex modeling environments in current tools, a clear, well organized model structure is very important to provide implicit documentation for users not intimately familiar with the design or model. Liberal use of comments and descriptions, while time consuming, is invaluable for documentation of the model.

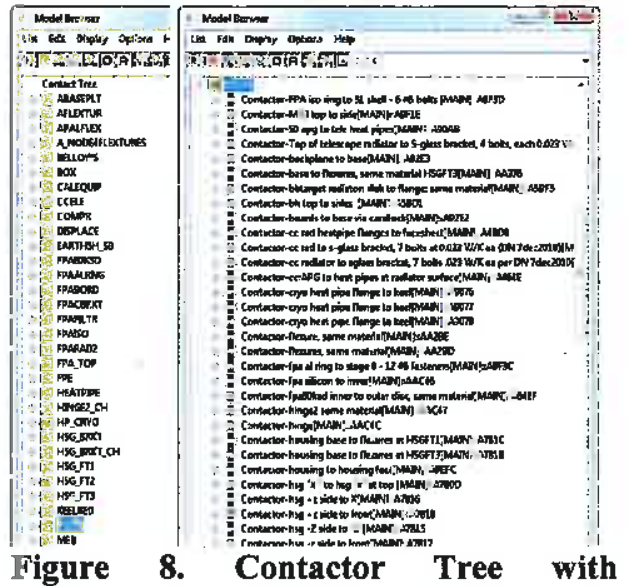

Submodels on left, Contactors in the MAIN Submodel on Right of the 243 total contactors, 90 were assigned to the MAIN submodel (37\%), making it difficult to determine which submodels were acted on by the contactor

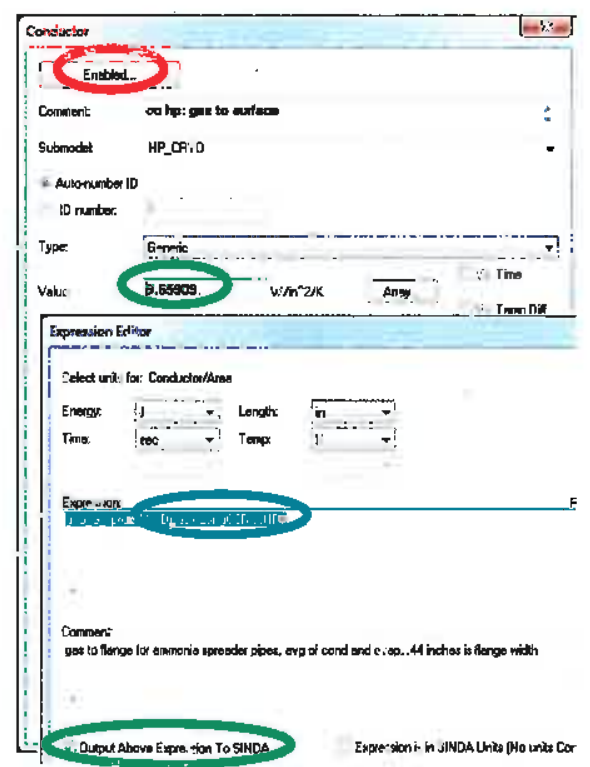

Figure 9. Conductor Form In Thermal Desktop Better to control value using symbol and outputting expressions than using Enable/ Disable entity for a run, since no reference is included in the thermal model if the entity is disabled at run time. Furthermore, it allows the state of the entity to change during a run, which enabling/disabling does not.

The necessity to perform numerous runs with various configurations and cases during the correlation and subsequent generation of final flight predictions necessitated a rapid turn-around of analysis. ThermalDesktop automatically takes advantage of multiple cores in modern PCs for the radiation ray tracing calculations, but the version of the thermal solver (SINDA/FLUINT 5.4) was a single threaded application and did not leverage the benefits of multiple cores. The latest version of SINDA/FLUTNT 5.5 does have some routines that take advantage of multiple cores within a single run, but this may not improve the overall throughput if multiple cases are run simultaneously. However, the configuration of multiple cases to only output the SINDA file, rather than output and execute, allowed for multiple runs to be executed externally in parallel through a master batch file. This master batch file called on secondary batch files for the serial execution and post processing of one particular case in its own command shell. 
The execution batch file created temporary folders to allow execution without conflicts with multiple runs trying to write to the same generic diagnostic files and moved the final result files back to the parent directory. Eight such calls from the master batch file allowed all the cores available on the analyst's PC to be utilized. Upon completion of the master batch file, generally over night, 8 or more cases were executed and the post-processed results were ready for further inspection the next day.

Lesson Learned: Execution of the thermal model outside of the ThermalDesktop environment can allow for faster completion of multiple cases by taking advantage of multiple CPU cores to run multiple cases rather than allowing ThermialDesktop to execute in a serial manner. Some care does need to be taken, since the current framework of SINDA is not configured to have multiple jobs running in the same subdirectory.

The pressure to present results as soon as they were available also resulted in some typographical analysis errors slipping through into results presented to the project. In one case, the wrong nodal temperature was reported for the EarthShield, masking a potential cold issue and limit violation in the cold safehold cases. Another typographical error for an $\varepsilon^{*}$ value of 0.3 instead of 0.03 indicated a significant problem with cooling of the telescope in test. Both of these errors were clerical in nature, but the disruption to productive work to move forward while the cause is investigated is not inconsequential when under schedule pressure. However, project insistence to have access to data prior to it being scrutinized and validated can allow these errors to escape the normal review and create manufactured issues that add stress to all parties involved. One area that could minimize some of these errors is the post processing of all the parametric values into a single comparable list to previous analysis cases. As models grow in complexity, with more and more capability and adjustments being able to be varied parametrically, the number of parametric terms (effective emissivities, power dissipations, heater setpoints, thicknesses, conductance values, etc.) grows significantly, making comparison to previously validated cases far more difficult. The data is readily available in a human readable format as comments within the generated thermal model input files, but comparing multiple files can be quite time consuming. A useful addition to the ThermalDesktop tool would be an improved comparison reporting capability, which would not only highlight the differences, but what values were different between cases.

Lesson Learned: Projects under extreme schedule pressure should consider the impact on the teams and that working faster can lead to more analysis mistakes than during the normal work flow pace. Careful checking of modeling parameters can help, but automated processes would greatly simplify the time consuming nature of comparisons.

\section{TIRS TV Test Execution Lessons Learned}

Accurate and thorough documentation of the test setup through photographs and notes is essential. All flight and test hardware was photographed and documented in electronic files that were referenced frequently during the correlation efforts when trying to compare model to actual hardware. All thermocouples and heaters were labeled such that the label was clearly visible in the pictures. It is also important to include enough surrounding hardware in the picture to be able to identify the location conclusively. Heater and circuit information such as size, resistance, location, etc. were recorded in spreadsheets and often referred to during real time troubleshooting. One specific instance where the photographs were of particular benefit was in the region of the EarthShield damper/hinge. A titanium bracket supports the damper and should provide significant thermal resistance between two sensors on the bracket assembly. However, test data indicated a very small temperature difference between the two sensors. Review of the photographs in this area and comparison to the model revealed an additional aluminum bracket that was added late in the design to support a survival heater and set of thermostats as shown in Figure 10. This additional aluminum bracket provided a low thermal resistance heat path in parallel to the high resistance titanium, but was never included in the model.

Lesson Learned: Proper documentation of all thermal test hardware is invaluable for real time troubleshooting and later for correlation.

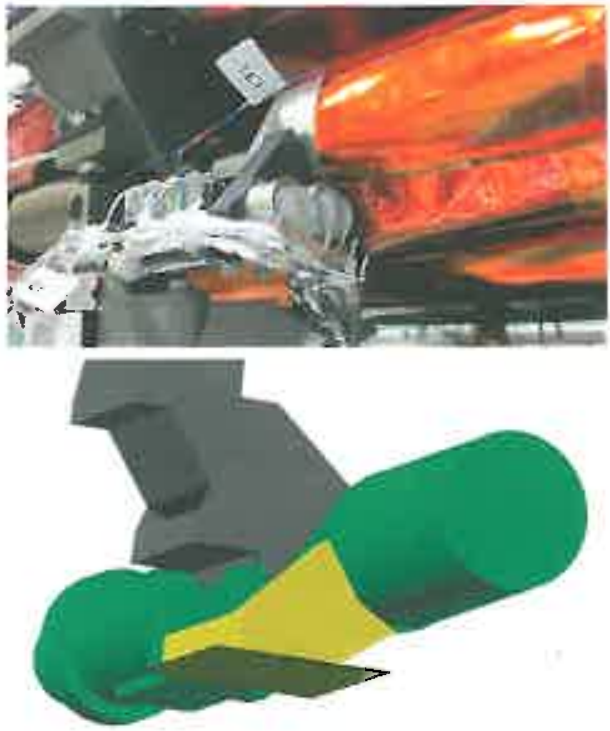

Figure 10. Damper Survival Heater Bracket The late addition of the aluminum bracket (yellow in model picture) created a high conductivity parallel path to the low conductivity hinge bracket 
During the test, the FPE temperatures were not in good agreement with the predictions. Eventually, this was traced to radiator area, but during the test this was not yet known. During troubleshooting, one area of question was the dissipation of the FPE, which was estimated, but not measurable with the available telemetry. The team identified a way to validate the dissipations using the flight heater with the box in an unpowered configuration to determine the additional heater power needed upon removal of the box internal dissipation. The FPE was allowed to reach steady state conditions in its operational state and the operational heater power recorded. As the box power was lowered from operational to standby and then again to off, the flight operational heaters increased in power to hold the temperature. After reaching steady state for each of these conditions, the increase in heater power was recorded. The calculated heater power is a good representation of the internal power since the heaters and card locks shared a common surface and thus the conductive path to the sink was the same. The flight operational heater calculation was well known and did verify that the power estimates provided were accurate. This method can be used with test heaters as long as the heat transfer path is similar and the heater power can be computed. A second clever use of heaters was used to determine the thermal conductance of the main harness to the instrument by modifying the settings for the zero-Q heater. The main power harness to these boxes was constructed with many wires and flexible shields. In flight, this cable originates from a room temperature FPE and connects to a sink temperature of approximately $253 \mathrm{~K}$. In order to get a check of this conductive path, the zero-Q heater was placed in temperature control mode and then turned off and the additional make up op power was recorded each time.

\section{Lesson Learned: Heaters can be used as to help determine important parameters such as internal power and measurement of important conductive paths.}

\section{TIRS Flight Design in Hindsight}

The knowledge gained during the Pre-PER and instrument level tests revealed some aspects in the TIRS design that if known prior to the design effort, would have likely resulted in a different design. The first of these is related to the cryocooler power discussed in previous sections. The high design dissipation imposed by systems due to the cryocooler dissipation uncertainty led to a much larger radiator than necessary, with about $70 \%$ of this radiator being blanketed in the final flight configuration, as shown in Figure 11. Furthermore, the aggressive schedule necessitated that procurement of the heatpipes and radiator begin prior to CDR, well before any measured cryocooler dissipations were available. With a limited surface area on the optimum $+Y$ side for heat rejection, the proportion of telescope radiator to cryocooler radiator could have been reapportioned to provide for more telescope radiator area and less cryocooler, if the actual dissipations had been known. Furthermore, the total areas used could also have been smaller, resulting in an overall reduction in the instrument size and corresponding mass.

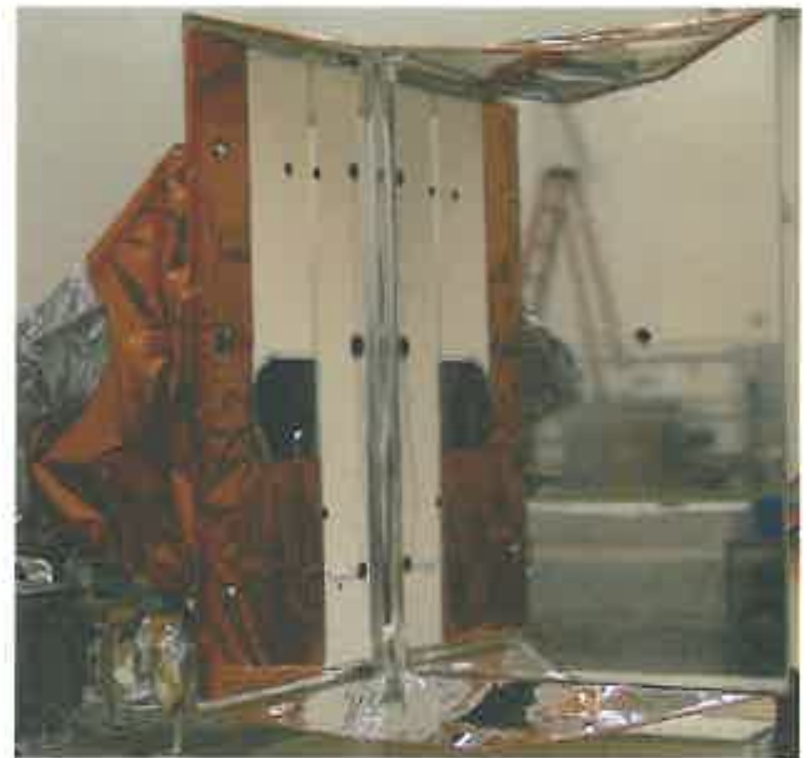

Figure 11. Final Radiator Configuration (Deployed) About $70 \%$ of the Cryocooler Radiator was blanketed for flight since the actual power was considerably less than the design value

The second area that would be redesigned knowing what is known now is the extensive use of titanium for the support structure for the Scene Select Mechanism (SSM). This housing features heaters on three circumferential sections of a cylindrical design to control the temperature of the bearings and motor. Unfortunately, the poor thermal conductivity of titanium, relative to a material like aluminum, induced significant gradients between heater locations and thermostats as well as along the axial direction of the housing. This housing was originally designed to support the telescope and therefore titanium was chosen to help provide the thermal isolation needed by the telescope. This design was later modified to provide independent isolation of the telescope from the mounting deck, but the SSM design was not revisited for its material selection of titanium, since procurement was already under way to meet schedule. At this point in the design flow, the design had matured and efforts to optimize the SSM were judged to be a significant enough impact to schedule. The consequence of this is that the heat dissipated by the heaters does not 
have a well designed path to leave the housing and as a result exits through numerous isolating paths. One noninsignificant path is across the bearings themselves, which results in a larger than expected gradient across the bearings, but within the design specification.

\section{Conclusions}

Even with a compressed schedule and significant pressure to deliver on time, the TIRS instrument completed instrument testing in January 2012 and was delivered in March of 2012 to the spacecraft. Further environmental testing at the spacecraft level from October 2012 through November 2012 presented no additional problems for TIRS and a successful launch commenced on February 11,2013 . While there is room for improvements of the design in hindsight, the instrument meets all the science requirements and is well poised to provide science data for the next 3 or more years as part of the Landsat Data Continuity Mission. During the development, testing, analysis and correlation, many lessons were learned focused on GSE design, pre-test analytical preparations, and modeling techniques to avoid or implement. For the design of GSE, it is recommended to carefully design the heater panels to have appropriate thicknesses, blanketing and heaters to minimize gradients. Prior to the test, it is important to ensure that the model accurately represents all the hardware, GSE included, and that all data necessary for correlation has been identified and that the model is capable of outputting these correlation parameters. Proper modeling includes good model organization and comments, avoiding large couplings to "short" two nodes together, appropriately simulating the physics of the design, and making use of modern computing resources to maximize run time efficiency. These lessons will be carried forward and applied to future missions at GSFC.

One last overarching lesson learned is that compressed schedules for the design, manufacture, and testing of spacecraft and instruments may result in successful designs, but it increases the risk of mistakes as well as placing stress and pressure on the teams involved. TIRS was successful due to the extensive commitments of many people working long hours tirelessly to meet the deadlines. At times, some corners were cut, but in the end the instrument was completed on time and resulted in a successful launch. However, the continuing success of missions with shortened time frames sets a dangerous precedent in that the compressed schedules may become the new "normal" without considering the extraordinary lengths gone through by the team to achieve success. Eventually, if schedules continue to be compressed, it is only a matter of time before the schedule has a direct link to a failure. TIRS is a successful project and will deliver valuable Earth science for years to come, but it should also serve as a potential warning about the unseen consequences of compressed schedules.

\section{Lessons Learned}

The lessons learned outlined in the paper are consolidated here into one single listing:

1. Be careful if partially blanketing heater panels as this could induce large gradients for thin panels. Careful pretest analysis should be used to determine an appropriate level of nodalization, optimum thickness, blanketing, and reasonable heater layout and placement to minimize gradients in the panel.

2. Unsupported internal closeout blankets can be very difficult to manewver into position and ensure that they do not move. If possible, provide a means of mechanically attaching the blanket and ensure the finished assembly is inspected.

3. Uncertainty in power dissipations can lead to a more complex test design that may be unnecessary. Projects should strongly consider the impacts on the entire program of simply carrying large design margins instead of ensuring that power dissipations can be measured within a reasonable time frame.

4. For aspects of a design where control authority is to be demonstrated by testing, it is recommended to conductively cold bias the temperature sink rather than relying on radiation and analysis, especially if the radiative cooling capability may be marginal.

5. Prior to testing, a thorough audit of a model should be performed by personnel (not just thermal) who are intimately familiar with the design and hardware to detect any discrepancies that could produce spurious predictions. This audit should include a mass check, size and dimension check, interface check, and actual radiator size verification. Model updates should be completed prior to beginning the test.

6. Prior to testing, a template should be developed with all the data parameters to be compared between model and test article (Flight Hardware Dissipations and Temperatures, GSE Dissipation and Temperatures, Configuration States, Heater Setpoints, etc). The model should also be configured to output all of the test data points for comparison. Doing this ahead of time will allow for the identification of gaps in the model or test data to be filled prior to the availability of the test as the test progresses. 
7. Prior to making any changes to the baseline pre-test model, generate a model with only the known values updated, such as power dissipation, GSE settings and temperatures. However, as a corollary to this recommendation, the baseline model should be configured prior to the test to be ready to accept these inputs without needing further modification. This same model should then be compared for Cold Balance and Hot Balance conditions to establish the pre-test model errors.

8. For cryogenic or near cryogenic designs, careful attention should be paid to the radiation couplings that are neglected as they could have importance if the temperature difference is sufficient.

9. Never couple two nodes with a very high coupling in order to make them have the same temperature. Renumber the node or merge them instead as this generates a far more robust thermal network for solution algorithms.

10. For cryogenic designs in particular, it is extremely important to include all sources of resistance in a heat path to avoid "shorting" sections which could result in overly optimistic predictions.

11. For the more complex modeling environments in current tools, a clear, well organized model structure is very important to provide implicit documentation for users not intimately familiar with the design or model. Liberal use of comments and descriptions, while time consuming, is invaluable for documentation of the model.

12. Use of 0 or 1 valued symbols as multipliers to nullify entity values is preferable to using symbols to enable or disable an entity for a run, since no reference is included in the thermal model if the entity is disabled at run time. Furthermore, it allows the state of the entity to change during a run, which enabling/disabling does not.

13. Execution of the thermal model outside of the ThermalDesktop environment can allow for faster completion of multiple cases by taking advantage of multiple CPU cores to run multiple cases rather than allowing ThermalDesktop to execute in a serial manner. Some care does need to be taken, since the current framework of SINDA is not configured to hove multiple jobs running in the same subdirectory.

14. Projects under extreme schedule pressure should consider the impact on the teams and that working faster can lead to more analysis mistakes than during the normal work flow pace. Careful checking of modeling parameters can help, but automated processes would greatly simplify the time consuming nature of comparisons.

15. Proper documentation of all thermal test hardware is invaluable for real time troubleshooting and later for correlation.

16. Heaters can be used as to help determine important parameters such as internal power and measurement of important conductive paths. 